\title{
Validation and reliability of translation of the ASAS Health Index in a Colombian Spanish speaking population with spondyloarthritis
}

\section{Author list}

Wilson Bautista-Molano',2, Robert B.M. Landewé ${ }^{3}$ Uta Kiltz', Rafael Valle-Oñate², Désirée van der Heijde'

' Rheumatology Department, Leiden University Medical Center, Leiden, The Netherlands

2Rheumatology Department, School of Medicine, Universidad Militar Nueva Granada and Rheumatology Department, Hospital Militar, Bogotá, Colombia

${ }^{3}$ Rheumatology Department, Academic Medical Center, University of Amsterdam, The Netherlands ${ }^{4}$ Rheumazentrum Ruhrgebiet, Herne, Germany

\section{Corresponding author:}

Wilson Bautista-Molano

Rheumatology Department, School of Medicine, Universidad Militar Nueva Granada

Transversal 3 No. 49-00 3" floor Bogotá, Colombia

wilson.bautista@gmail.com 


\section{ABSTRACT}

Objective To validate a Spanish-language translation of the ASAS Heath-Index (ASAS-HI) testing its reliability, construct validity and responsiveness in Colombian-patients with spondyloarthritis.

Methods Translation was done following a forward-backward procedure. Patients fulfilling the ASAS criteria for either axial or peripheral-SpA participated. Test-retest reliability was assessed by intraclass correlation coefficient (ICC) in patients without treatment changes. In patients who required a therapeutic intervention, responsiveness was assessed using the standardized response mean (SRM). Construct validity was evaluated by Spearman correlation. Internal consistency (Cronbachs- $\alpha$ ) and discriminative ability of the ASAS-HI were assessed.

Results Fifty patients were included: 54\% male, mean (SD) age 44.8(13.1), symptom duration 15.8(9.7) years, BASDAI 4.6(2.2), BASFI 4.7(2.5), ASDAS-CRP 2.2(1.0). AxSpA was established in 44 patients $(\mathrm{AS}=30, \mathrm{nr}-\mathrm{axSpA}=14)$ and $\mathrm{pSpA}$ in 6. The score of the ASAS-HI was 8.2(5.1). The test-retest reliability was good with an ICC of 0.84 . SRM was 2.58 (1.75-3.37) in 10 patients with any intervention and 2.94 (2.13-4.24) for 7 patients starting TNF-blockers. Construct validity showed a good correlation between ASAS-HI and pain, BASDAI, BASFI, and ASDAS ( $r \geq 0.60)$. A high internal consistency was found with a Cronbachs- $\alpha$ of 0.91 . ASAS-HI discriminated well between patients with different stages of disease activity (BASDAI and ASDAS). Those with higher disease activity had higher ASAS-HI scores. Conclusion The Spanish-language translation of the ASAS-HI has proven to be psychometrically valid for Colombian-patients with SpA. This version is available to evaluate the state of health and functioning in these patients and can be used in clinical practice.

Key words: Spondyloarthritis, health, functioning 


\section{INTRODUCTION}

The term spondyloarthritis $(\mathrm{SpA})$ is used to describe a disease characterized by axial and entheseal inflammation, as well as extra articular manifestations such as uveitis and psoriasis [']. Ankylosing spondylitis (AS) is the prototype of SpA and together with non-radiographic axial SpA (nr-axSpA), constitutes the subgroup that is termed axial SpA (axSpA). This group of patients differentiates from peripheral $\mathrm{SpA}(\mathrm{pSpA})$ by the predominance of axial- rather than peripheral manifestations as presenting feature $\left.{ }^{\left[{ }^{2}\right.}\right]$. Given the variable course of $\mathrm{SpA}$, the evaluation of health and functioning is increasingly recognized by health care professionals to be an important outcome when assessing the impact of the disease on the patient.

The Assessment of Spondyloarthritis international Society (ASAS) Health Index (HI) has been developed to measure functioning and health in patients with $\mathrm{SpA}$ aiming to better describe the impact of disease in these patients [']. The ASAS-HI is a linear composite measure based on an item pool which has been developed and based on the International Classification of Functioning (ICF) core set for AS [?]. This index forms a unidimensional scale providing a sum score representing a wide spectrum of different levels of functioning and contains 17 dichotomous aspects addressing the following categories: pain, emotional functions, sleep, sexual functions, mobility, self-care, community life and employment. The questions of the questionnaire range from a score of 0 to 17, with a lower score representing a better health status. Recently, the ASAS-HI has been translated and adapted cross-culturally into 15 languages worldwide [ ${ }^{6}$.

The English version has proven to be valid and reliable for use in patients with SpA [?] [8]. Since validated Spanish patient-reported and composite measures do not exist for assessing the broad impact of health 
and functioning in SpA patients, the present study aimed to translate and cross-culturally adapt the English version of the ASAS-HI into Spanish-language spoken in Colombia and to re-assess reliability and construct validity for patients with SpA after translation.

\section{METHODS}

\section{Translation and cultural adaptation}

Translation and cross-cultural adaptation of the English version of ASAS-HI was performed according to the published recommendations using a standardized operating procedure (forward-backward procedure) [']. This method consists of the following 5 steps: translation, synthesis of translation, back translation, expert committee review and pre-testing in a field test with cognitive debriefing. First, three persons (all bilingual) with different profiles or background (including non-medical), independently performed a forward translation of the instrument from the original language (English) to the Spanishlanguage according to the characteristics of our patients. Back translation was then conducted by two independent bilingual native English speakers blinded to the English version. The expert committee agreed upon the final wording of the Spanish version, who examined semantic, idiomatic, experiential and conceptual issues. All the versions of the questionnaire were consolidated to develop a pre-final version for the field test. The final version (Appendix 1) was tested with 10 native patients with axSpA (60\% AS and $40 \% \mathrm{nr}-\mathrm{axSpA})$. The patients completed the questionnaire in a face to face interview with a physician. Each question was discussed with the patient in order to check whether all items had been fully understood and whether the patient had problems with wording, relevance, acceptability, incomplete concept coverage and comprehensiveness of the translation. Notes were taken about the time to complete the questionnaire and whether the patients made comments about specific items when completing the questionnaires. 


\section{Participants}

A sample of patients from a rheumatology outpatient clinic in Colombia were invited to complete the ASAS-HI. Adult SpA patients fulfilling the ASAS classification criteria either axial or peripheral were eligible for participation. According to the ASAS international validation study ${ }^{6}$ a convenient sample was obtained: at least $80 \%$ of patients with axSpA and a maximum of $20 \%$ with pSpA. Estimated proportion of axSpA patients were approximately $40 \%$ with nr-axSpA and $60 \%$ with AS. The institutional ethics committee approved the study. Informed consent was obtained from all participating patients.

\section{Assessments}

Demographic and clinical information was collected in the first visit (baseline) including age, gender, years of formal education, work status, presenting symptoms, extra articular manifestations and current results of laboratory test for C-reactive protein (for ASDAS) [ $\left.{ }^{\circ}\right]$. To assess the different disease aspects of health, patients were asked about their disease duration (symptoms duration), current medication and completed the patient global assessment (NRS), pain (NRS), spinal pain (NRS), the Bath Ankylosing Spondylitis Disease Index (BASDAI) ["], the Bath Ankylosing Spondylitis Functioning Index (BASFI) ${ }^{[2]}$, EuroQol five dimensions questionnaire (EQ-5D) [ ${ }^{13}$, Short Form Survey Instrument 36-Item (SF-36)

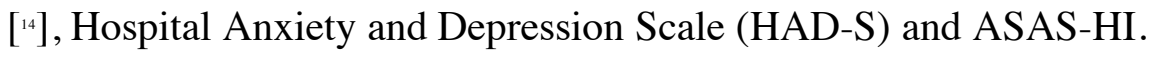

\section{Reliability}

A subsample of patients with no change in treatment (no change in NSAIDS over last week, no change in DMARD or TNF blockers over last 4 weeks) who considered themselves in a stable disease state were 
invited to complete the questionnaire at home after 4-7 days interval to evaluate reproducibility (reliability arm).

Sensitivity to change

Patients with clinical disease activity who required an important therapeutic intervention because of unacceptable clinical disease activity were invited to complete the questionnaire during a second visit 424 weeks after the initiation for NSAIDS and 12-24 weeks after the initiation for DMARDS (methotrexate, sulfasalazine) or TNF blockers (sensitivity arm). Baseline assessments including the questionnaires and clinical assessments were performed in the clinic.

\section{Statistical analysis}

Reliability was assessed by the test-retest method at 4-7 days interval for patients who considered themselves in a stable state. It was measured using the intra-class correlation coefficient (ICC) as an estimate of the instrument reproducibility over time -assuming that no modification in health conditions had occurred. For testing sensitivity to change, patients were included in the analyses if they report improvement on a global change question. Results obtained before and after treatment were compared and sensitivity to change was measured using the standardised response mean (SRM), which is calculated by dividing the mean score change by the standard deviation (SD) of the change.

Construct validity was analysed between ASAS-HI score and several other health outcomes: patient global, pain, spinal pain, BASDAI, BASFI, ASDAS, SF-36, EQ-5D and HAD-S using Spearman's correlation coefficient (considered good if $\geq 0.6$ ). Internal consistency was evaluated by Cronbach's $\alpha$ coefficients (adequate: $\geq 0.70$ ) $\left[{ }^{15}\right]$. 
The discriminant ability of the ASAS-HI was assessed between groups differing in overall health -the four disease activity states according to the ASDAS score [ ${ }^{10}$ (inactive, low, high and very high). The difference between the four ASDAS groups were tested by analysis of variance. All statistical tests were two-sided and $\mathrm{p}$ values $\leq 0.05$ were considered significant. Statistical analyses was done using SPPS (Chicago, Illinois, USA) version 19.

\section{RESULTS}

\section{Translation and cultural adaptation}

No major problems for translation and cross-cultural adaptation were observed. Overall, none of the patients had difficulties in answering the ASAS-HI and they felt comfortable with evaluating the questionnaire. Results of the cognitive debriefing demonstrated that the translated version of the ASAS-HI was properly understood. The mean (SD) time to complete the ASAS-HI was 2.55 (0.9) minutes.

\section{Clinimetric properties of the ASAS-HI}

Subjects

Fifty patients agreed to participate and completed the questionnaire. The diagnosis was axSpA in 44 patients: AS $(n=30) n r-a x S p A ~(n=14)$ and $p S p A$ in 6 patients. Of these patients, 18 participated in the test-retest reliability study arm and 10 participated in the sensitivity to change study arm. The characteristics of the population are presented in table 1. Fifty four percent of the patients were male, mean age of 44.8 (13.1) years and symptom duration of 15.8 (9.7) years. The mean scores for clinical assessments were: BASDAI 4.6 (2.2), BASFI 4.7 (2.5) and ASDAS-CRP 2.2 (1.0). The total score of the ASAS-HI was $8.2(5.1)$. 
Test-retest Reliability

The mean (SD) baseline ASAS-HI was 8.9 (5.0) and the second ASAS-HI was $7.4(5.4)$ in the 18 patients in the reliability arm. The ICC was good 0.84 (95\% CI 0.71 to $0.93, \mathrm{p}<0.001)$.

Sensitivity to change

Ten patients required change of treatment: 7 patients started a TNF blocker, 2 patients started NSAIDS and 1 patient a DMARD. The SRM was 2.58 (1.75 to 3.37) for all patients requiring modification of treatment $(n=10)$. For those patients in which the intervention was starting a TNF blocker $(n=7)$ the SRM was higher: 2.94 (2.13 to 4.24$)$ than those starting a NSAID or DMARD (n=3), in which the SRM was $2.22(1.23$ to 3.21$)$.

Construct validity

The ASAS-HI had good correlation with the following disease specific clinical parameters: patient global $(r=0.58)$, spinal pain $(r=0.59)$, pain, BASDAI, BASFI, ASDAS, and HAD-S $(r=0.60-0.70)$ and the highest correlations with EQ-5D and SF-36 ( $>0.70)$. The correlations of ASAS-HI with age and symptom duration were weak. Results are presented in table 2.

Internal consistency

ASAS-HI scores showed a high internal consistency with a Cronbach's alpha of 0.91 . 
Discriminative ability

Finally, the ASAS-HI was discriminative between the four health states of the ASDAS score (inactive, low, high and very high disease activity). The groups with greater disease activity had higher mean ASAS HI scores than those with lower disease activity $(\mathrm{p} \leq 0.001)$. Results are given in table 3 .

\section{DISCUSSION}

The impact of SpA in many aspect of life in patients having this condition is considerable. Many aspects related to the burden and severity of the disease such as disease activity, physical function, social participation and work ability, should be considered when assessing patients with SpA. Moreover, the evaluation of health ${ }^{\left.{ }^{16}\right]}$ and functioning is increasingly recognized as an important outcome in order to evaluate the impact of the disease on the patient. With this regard, the ASAS-HI was developed and is available as a useful tool to measure functioning and health in patients with SpA

The translated and culturally adapted version of the ASAS-HI reported in the current study maintains all the properties of the original English-language version of the questionnaire. The procedure implemented to translate the instrument followed a standardized operating procedure according to the recommendations published by Beaton [ $\left.{ }^{\circ}\right]$. Further we tested several aspects of its validity, relevance and comprehensibility among patients with SpA. Therefore, the health status and functioning in Spanishspeaking patients with SpA may be properly assessed with the translated version of the original ASASHI.

The psychometric properties of this Spanish-language version of the ASAS-HI were consistent with the results found in the original study [ ${ }^{6}$, and also with the study of the validity of the Korean-language 
translation ${ }^{\left[{ }^{17}\right.}$. Similarly, an Italian study also have confirmed the reliability, feasibility and validity of the ASAS-HI $\left.{ }^{18}\right]$. The reliability of the adapted version showed a good test-retest reliability $($ ICC $>0.8)$ and a high internal consistency (Cronbach- $\alpha>0.9)$. The ASAS-HI was sensitive to pick up changes after starting a new pharmacological treatment. The SRM was higher than those seen in the original description of the instrument, a fact that could be explained by the higher baseline values on the instrument that could imply a higher probability of significant changes after effective treatment. Regarding construct validity, the translated version had good correlation $(>0.6)$ with several clinical parameters used currently to assess function, disease activity and quality of life in patients with SpA. In addition, the instrument was discriminative regarding different thresholds of disease activity.

Some limitations need to be considered. First, when evaluating test-retest reliability, no external and objective measure was included to assess stability of the health condition. However, it is accepted that a seven-day period in a chronic disease is an appropriate interval to avoid a significant variability in health, on the one side, and avoid too much recall bias on the other side. Second, the Spanish-language version of the ASAS-HI validated in the current study is validated for the Colombian population. Regarding other Spanish-speaking populations, the use of this version may require additional cross-cultural adaptation and validation mainly due to linguistic variations and cultural differences. Two additional Spanish-versions of the ASAS-HI are available (Mexico and Spain) and were elaborated independently during the international validation project. The differences with these translated tools are restricted to a few changes in wording. For instance, the original English version asks about problems using toilet facilities. The word "toilet" was translated using different Spanish words in each country. In the same way, "exhausted" was translated using three different words having the same meaning. Similar findings were observed with the translation of the following words: "car", "hair" and "flat ground". Although 
these semantic differences may reflect the transcultural adaptation of the instrument in each country, the underlying concept to assess functioning remains the same. Third, although the 9 items of the Environmental Factors sets related to the ASAS-HI were additionally translated and evaluated in the field test, the questionnaire was not included in the current study.

Our study contributes to $\mathrm{SpA}$ assessment by providing a reliable and valid cross-culturally adapted Spanish-speaking version of the ASAS-HI that is sensitive to change. This adapted instrument may be complementary to previous tools validated in Spanish-language and currently implemented in trials and clinical practice to assess patients with $\operatorname{SpA}\left[{ }^{19},{ }^{20}\right]$.

In conclusion, the Spanish ASAS-HI version represent a relevant and comprehensive patient-based instrument for evaluating the state of health and functioning in Colombian patients with SpA. This version is available and can be used in research and in clinical practice.

Diclosures: All authors declared no conflict of interests.

Wilson Bautista-Molano: none

Robert Landewé: none

Uta Kiltz: none

Rafael Valle-Oñate: none

Désirée van der Heijde: none 


\section{REFERENCES}

Dougados M, Baeten D (2011). Spondyloarthritis. Lancet 377:2127-37

2 Rudwaleit M, van der Heijde D, Landewé R, et al (2009). The development of Assessment of SpondyloArthritis international Society classification criteria for axial spondyloarthritis (part II): validation and final selection. Ann Rheum Dis 68:777-83

${ }^{3}$ Rudwaleit M, van der Heijde D, Landewé R, et al (2011). The Assessment of SpondyloArthritis International Society classification criteria for peripheral spondyloarthritis and for spondyloarthritis in general. Ann Rheum Dis 70:25-31

${ }^{4}$ Kiltz U, van der Heijde D, Boonen A, et al (2015). Development of a health index in patients with ankylosing spondylitis (ASAS HI): final result of a global initiative based on the ICF guided by ASAS. Ann Rheum Dis 74:830-5

${ }^{5}$ Boonen A, Braun J, van der Horst Bruinsma IE, et al (2010). ASAS/WHO ICF Core Sets for ankylosing spondylitis (AS): how to classify the impact of AS on functioning and health. Ann Rheum Dis 69:102-7

${ }^{6}$ Kiltz U, van der Heijde D, Boonen A, et al (2016). Measuring impairments of functioning and health in patients with axial spondyloarthritis by using the ASAS Health Index and the Environmental Item Set: translation and cross-cultural adaptation into 15 languages. RMD Open 2:e000311. doi:10.1136/rmdopen-2016-000311

${ }^{7}$ Kiltz U, van der Heijde D, Boonen A, et al (2014). The ASAS Health Index (ASAS HI) - a new tool to assess the health status of patients with spondyloarthritis. Clin Exp Rheumatol 32:S-105-8 ${ }^{8}$ Kiltz U, van der Heijde D, Boonen A, et al. (2018). Measurement properties of the ASAS Health Index: results of a global study in patients with axial and peripheral spondyloarthritis. Ann Rheum Dis Jun 1 pii: annrheumdis-2017-212076. doi: 10.1136/annrheumdis-2017-212076

' Beaton DE, Bombardier C, Guillemin F, et al. (2000). Guidelines for the process of cross-cultural adaptation of self-report measures. Spine 25:3186-91

${ }^{10}$ Machado P, Landewé R, Lie E, et al (2011). Ankylosing Spondylitis Disease Activity Score (ASDAS): defining cut-off values for disease activity states and improvement scores. Ann Rheum Dis 70:47-53

"Garrett S, Jenkinson T, Kennedy LG, et al (1994). A new approach to defining disease status in ankylosing spondylitis: the Bath Ankylosing Spondylitis Disease Activity Index. J Rheumatol 21:2286-91

${ }_{12}$ Calin A, Garrett S, Whitelock H, et al (1994). A new approach to defining functional ability in ankylosing spondylitis: the development of the Bath Ankylosing Spondylitis Functional Index. J Rheumatol 21:2281-5

${ }^{13}$ Brooks, R. (1996) EuroQol: the current state of play. Health Policy 37:53-72

${ }^{14}$ Ara, R. and Brazier J (2008). Deriving an algorithm to convert the eight mean SF-36 dimension scores into a mean EQ-5D preference-based score from published studies (where patient level data are not available). Value Health 11:1131-43

${ }^{15}$ Terwee CB, Bot SD, de Boer MR, et al (2007). Quality criteria were proposed for measurement properties of health status questionnaires. J Clin Epidemiol 60:34-42

${ }^{16}$ Salaffi F, Carotti M, Gasparini S, et al (2009). The health-related quality of life in rheumatoid arthritis, ankylosing spondylitis, and psoriatic arthritis: a comparison with a selected sample of healthy people. Health Qual Life Outcomes 18;7:25 
"Choi JH, Kim TJ, Shin K, et al (2014). The reliability and validity of a Korean translation of the ASAS Health Index and Environmental Factors in Korean patients with axial spondyloarthritis. J Korean Med Sci 29:334-7

${ }^{18}$ Di Carlo M, Lato V, Carotti M, et al (2016). Clinimetric properties of the ASAS health index in a cohort of Italian patients with axial spondyloarthritis. Health Qual Life Outcomes 14:78

${ }^{19}$ Cardiel MH, Londoño JD, Gutiérrez E, et al (2003). Translation, cross-cultural adaptation, and validation of the Bath Ankylosing Spondylitis Functional Index (BASFI), the Bath Ankylosing Spondylitis Disease Activity Index (BASDAI) and the Dougados Functional Index (DFI) in a Spanish speaking population with spondyloarthropathies. Clin Exp Rheumatol 21:451-8

${ }^{20}$ Ariza-Ariza R, Hernández-Cruz B, Navarro-Sarabia F. (2003). Physical function and healthrelated quality of life of Spanish patients with ankylosing spondylitis. Arthritis Rheum 49:483-7 